\title{
Temperament and Visual Category Learning Strategy Use
}

\author{
Tianshu Zhu', John Paul Minda ${ }^{\mathrm{a}}$ \\ ${ }^{a}$ The University of Western Ontario
}

\begin{abstract}
Transient affective states have been shown to affect cognition, including category learning, but less is known about the role of stable temperament traits and categorization. We examined affective temperament traits to see whether the tendency to experience negative and positive affect is predictive of category learning performance and strategy use. Working memory and attentional control were measured as covariates. Participants first completed the Adult Temperament Questionnaire (Evans \& Rothbart, 2007) including two affective temperament factors and an attentional control factor. Then they completed a memory task followed by either a conjunctive rule-based (CR) or an information integration (II) category learning task. Results showed that people who tend to experience more positive affect and less negative affect achieved higher accuracy and were more likely to find the optimal strategy in the II task compared to people who tend to experience more negative affect and less positive affect. However, no performance or strategy use difference was seen in the CR task across different temperament profiles. These results extend prior literature and provide additional insights on the effects of stable traits on category learning.
\end{abstract}

Keywords: categorization, temperament, learning, cognitive modeling

\section{Introduction}

Categorization is a fundamental phenomenon of cognition and intelligent behavior. The mental representations of categories are called concepts and these are the core building blocks of the knowledge we have about the world. In other words, understanding categorization behaviors is the first step to understanding cognitive processes. Categorization is not only critical to our day-to-day activities (e.g., identify food items from non-edible items), it is also a learned skill that is heavily involved in many professional fields such as radiology (Hatala et al., 2003) and forensics (Searston \& Tangen, 2017). Understanding how people group objects together is an essential step in identifying ways to enhance the learning process and enable better categorical knowledge transfer or generalization. Knowledge transfer is the ultimate purpose of categorization, which allows us to make implications based on the category membership and react accordingly without having to experience every single object. Category learning is a model-rich field, its main emphasis has been on developing computational models to account for people's strategic approach in their categorization responses. The goal of the current study is to explore the ways in which individual differences in temperament can lead to variations in category learning strategy use. 


\section{Dual-process of Category learning}

Steven Sloman (1996) proposed a version of dual-process theory within the judgment and decision making literature. This account followed a parallel-competitive architecture -- the associative (automatic) and rule-based (analytic) processing occur in parallel and compete to find the solution. A number of related dual-process models have also been proposed in category learning research (Anderson \& Betz, 2001; Ashby et al., 1998; Bott \& Heit, 2004; Erickson \& Kruschke, 1998). These models contend that categories can be learned either through an explicit rule-based system or an implicit similarity-based system. One of the most researched multiple systems models of category learning is the competition between verbal and implicit systems (COVIS) model. It postulates that the explicit and implicit systems compete throughout learning, much aligned with Sloman's account. The explicit system requires hypotheses testing of verbalizable rules and effortful working memory engagement, whereas the implicit system operates effortlessly and is associated with motor learning (e.g., Ashby et al., 1998). Compared to the consensus with regards to the nature of the explicit system, there have been debates around whether the implicit system relies on exemplar-based memorization (e.g., Brooks, 1978; Estes, 1986; Medin \& Schaffer, 1978; Nosofsky, 1986), prototype abstraction (e.g., Homa et al., 1981; Minda \& Smith, 2001; Reed, 1972) or procedural-based cognitive mechanisms (e.g., Ashby \& Gott, 1988; Ashby \& Lee, 1991; Ashby \& Maddox, 1990; Ashby \& Maddox, 1992, 1993).

Evidence supporting the dissociation between the explicit and implicit systems has mostly come from category learning tasks whose stimuli and structures can be best learned through either an explicit process or an implicit process (cf. Erickson, 2008). These tasks originated from the decision bound theory which assumes that subjects learn to associate category space partitioned by the decision bound(s) with particular category responses (Ashby \& Gott, 1988; Ashby \& Maddox, 1993; Ashby \& Townsend, 1986). Certain decision bounds are easily verbalizable in relation to stimulus dimensions, resulting in the development of rule-based category structures. Other decision bounds cannot be easily described in terms of stimulus dimensions, resulting in the development of similarity-based category structures. These similarity-based categories can be learned by attending to holistic similarities among category members. Many studies have used explicit and implicit category structures based on the decision-bounds and found dissociations between the two types of structures in terms of the underlying process that mediated optimal learning (Ashby et al., 1999; Ashby et al., 2002, 2003; Ashby \& Waldron, 1999; Maddox et al., 2003; Smith et al., 2014). Dissociation between the explicit and implicit systems is evident when differential behavioural outcomes were elicited in the rule-based and similarity-based categorization tasks by the same experimental manipulation. For instance, Ashby et al. (2002) showed that training type affected the similarity-based category structure much more than the rule-based category structure. Smith et al. (2014) found that deferred feedback significantly impacted similarity-based learning but spared rule-based learning.

Interestingly, despite the average patterns described above, some studies have shown that individuals may differ in their strategic approach in learning either the rule-based or similarity-based tasks (Donkin et al., 2015; Minda \& Smith, 2001; Pelley et al., 2019). For example, a subgroup of participants may show a strong tendency or preference for rule use and apply this strategy to a similarity-based task despite having lower performance accuracy than people who used the task appropriate strategy. Understanding the individual factors that are associated with suboptimal category learning is the first step towards identifying ways to promote optimal performance and effective knowledge transfer. In this study, we used a rule-based and a similarity-based category learning task to detect people's strategy use tendency, 
and to examine whether this tendency is related to the few individual differences variables we discussed in the next sections.

\section{Factors that Influence an Individual's Learning Strategy}

\section{Executive Attention and Working Memory Capacity}

Prior research has shown that age-related changes in cognition can impact individuals' performance and strategy use on rule-based and similarity-based category learning tasks. Rabi and Minda (2016) showed that older adults performed significantly worse than young adults on both the task that required discovery of a complex rule (disjunctive rule-based; Type II category of SHJ task) and the task that required similarity-based processing (information integration; Type IV category of SHJ task). Older adults tended to rely more on suboptimal single-dimensional rule in Type II category, and they were less consistent in applying the similarity-based strategy in Type IV category. Maddox et al. (2010) also found that normal aging impacts both types of category learning and this may be resulted from diminished inhibitory control. Along the same line, Racine et al. (2006) found that older adults performed on par with younger adults on the single-dimensional rule-based task, but showed deficits when the rule became complex. Minda and colleagues (2008; Rabi \& Minda, 2014) showed that young children similarly showed deficits in learning more complex rule-based strategies, but their performance did not differ from young adults in learning simple rules and similarity-based strategies. Studies have compared young children and adults' strategy-use in learning ambiguous categories and found that children relied mostly on overall similarity, while adults tend to rely on the deterministic rule (Deng \& Sloutsky, 2016; Miles \& Minda, 2009). These studies showed that young adults have an advantage learning complex rule-based categories than young children and older adults, but the learning advantages were less consistently observed in similarity-based tasks.

These observed behavioral differences can be attributed to neurophysiological changes across the lifespan. Neuroimaging studies have shown that rule-based cognitive tasks engage the prefrontal cortex, and task-related activations in the head of the caudate nucleus and the anterior cingulate have also been reported (Konishi et al., 1999; Lombardi et al., 1999; Rao et al., 1997; Rogers et al., 2000; Volz et al., 1997). These are critical components of the structures relating to working memory (Gabrieli, 1995; Goldman-Rakic, 1995; Goldman-Rakic, 1995) and the executive attention system (Miller \& Wickens, 1991; Posner \& Petersen, 1990). Executive attention is considered to be a component of working memory by Engle and colleagues, this component is defined as the ability to select and maintain task goals and avoid distraction (Engle \& Kane, 2004). The other component of working memory is storage (Baddeley, 2012). Corbetta et al. (1991) suggest that the above-mentioned brain structures mediate rule-based category learning. The prefrontal cortex along with other areas underlying executive attention and working memory takes many years to fully develop during normal growth, therefore, young children (e.g., younger than 5 years old) often have difficulty with selectively attending to the relevant stimulus dimension(s) when testing or applying a rule (Hanania \& Smith, 2009; Plude et al., 1994), as well as with remembering and shifting attention to untested rules (Aschkenasy \& Odom, 1982; Gholson et al., 1972; Kemler, 1978). The deficits seen in older adults can also be attributed to diminishing activities in the structures related to executive attention and working memory. Older adults had more difficulty than young adults with resolving conflicts within tasks and inhibiting task-irrelevant behaviors (Davidson et al., 2003; May \& Hasher, 1998; West, 1999; Williams et al., 1999), which demonstrated compromised executive attention. The decline in working memory is also evident in older adults, reflected both through verbal and spatial aspects (Bopp \& Verhaeghen, 2005; Park et al., 2002). 
Studies that examined the effects of executive attention and working memory on category learning using only young adult participants have generally shown that higher capacities are associated with more task appropriate strategy use. Lewandowsky et al. (2012) showed that a single latent variable captured the variation in learning across many different types of categorization tasks, and this latent variable was associated with working memory capacity. This suggests that working memory capacity mediated the learning of all types category structures regardless of the task appropriate strategy. Similarly, Kalish et al. (2017) found that individuals with higher working memory capacity tended to perform more accurately on both analytic-process and procedural-process mediated category learning tasks. McDaniel et al. (2014) used a variety of category generalization tasks to investigate whether an individual's tendency to use explicit or implicit processes was related to a number of cognitive capacities. They found a positive relationship between working memory and the tendency to use rules. One thing to note here is that the tasks in McDaniel et al.'s study can be learned equally well using the rule-based or similarity-based strategy, so it was different from the other reviewed studies that generally promoted a superior task appropriate strategy. Therefore, McDaniel and colleagues measured the direct relationship between cognition and strategy-use tendency, without participants' responses being swayed by biased feedback. These results do not necessarily contradict with other reviewed studies which suggest that when an optimal strategy is provided, high executive attention and working memory capacity tend to facilitate task appropriate learning.

Both developmental research and studies that recruited only young adults suggest that both higher executive attention and higher working memory capacity are associated with an advantage in learning complex rule-based categorization tasks, but results on how these cognitive capacities affect similarity-based tasks were less consistent.

\section{Emotion or Affect}

\section{Category Learning}

Ashby and colleagues (Ashby et al., 1998, 1999) proposed the dopaminergic theory of positive affect, which argues that the effects of positive affect on cognition are mediated by dopamine. It also assumes that increase of dopamine release in the anterior cingulate during transient positive mood states increases cognitive flexibility and verbal fluency. Ashby and colleagues suggest that positive affect facilitates explicit rule-based learning and implicit similarity-based learning. Cognitive flexibility and verbal fluency are related to rule selection and rule switching (Owen et al., 1993), thus improving the efficiency of discovering the optimal strategy. The dopaminergic theory further postulates that striatal dopamine is critical in mediating feedback in procedural learning (Ashby et al., 2007). Positive affect facilitates similarity-based tasks through projecting dopamine into the striatum, a critical region involved in this type of processing. Moreover, cognitive flexibility may also speed up the process of exhausting potential rule-based strategy before resorting to the similarity-based strategy. Based on the dopaminergic theory, positive affect should facilitate both rule-based and similarity-based category learning, while negative affect should undermine these learning processes. Empirical evidence has supported the dopaminergic theory on its assumption that positive mood facilitates rule-based category learning, but results were less consistent with regard to negative affect as well as similarity-based category learning. Nadler et al. (2010) found that positive mood facilitated optimal strategy discovery on both rule-based and similarity-based category learning tasks, but significant performance difference between mood states was only observed in the rule-based task. Nielsen and Minda (2018) found positive mood significantly facilitated learning on the rule-based categorization task, but they did not find any mood effects on learning of the 
similarity-based task. Ashby et al. (1998) showed that clinically depressed individuals suffer from rule-based learning deficits.

On the other hand, results contradicting the dopaminergic theory are also abundant. (Noseworthy \& Goode, 2011) looked at the mood effects on people's tendency to use heuristic approach (i.e., criterial inferencing) in categorizing consumer products, and they found positive mood led to more biased responses while negative mood promoted more analytic evaluation. Gorlick \& Maddox (2013) manipulated participants' mood and found that negative arousal had facilitatory effects on rule-based prototype learning, and detrimental effect on similarity-based prototype learning, vice versa for positive arousal. To further complicate the picture, Maddox et al. (2014) found that elevated depressive symptoms facilitated the learning of an similarity-based auditory category learning task. This advantage was shown through earlier adoption of optimal implicit strategy and more accurate application of these strategies by individuals with elevated depressive symptoms than healthy controls.

As we can see, there is no single pattern with respect to the mood effects in category learning literature, it is reasonable to resort to a broader literature that examines mood effects on cognition in general.

\section{Social cognition}

Before reviewing relevant studies, it would be helpful to understand the relationship between rule-based/similarity-based categorization strategies and local-analytic/global-holistic processing. In a typical rule-based category learning task, learners need to identify and attend to the only relevant stimulus dimension (the rule), and then map each dimension value to different category labels (Ashby \& Todd Maddox, 2005). Narrowing attention to specific details is synonymous to local processing (Kimchi, 1992; Navon, 1977) and formulation of an explicit verbal rule relies on analytic resources (Hayes-Roth, 1985). This is not to say that rule-based category learning tasks can only be single-dimensional: conjunction and disjunctive rules are also viable options, and these more complex rules also require analytical processing of relevant stimulus dimensions. On the other hand, similarity-based category learning requires learners to combine several stimulus dimensions to derive at a category decision, and the stimulus often needs to be processed as a gestalt (Ashby et al., 1998). Not surprisingly, this information integration approach overlaps closely with global holistic processing and procedural learning (Navon, 1977; Kimchi, 1992). Following from these, it can be reasoned that explicit rule-based strategies are related to local and analytic processing, while implicit similarity-based strategies are related to global and holistic processing.

One prominent theory that tries to explain the association between affect and processing style is the affect-as-information hypothesis (Clore, 1992; Schwarz \& Clore, 1983), which proposes that people rely on their mood to judge the nature of the environment. Positive affect would signify that the environment is safe, therefore people tend to engage in heuristic and holistic processing; if the negative mood was prominent, then people would switch to a more analytical processing style to assess the problematic environment. Proponents of this view have shown that the mood effects often disappear when one's feelings were rendered nondiagnostic (Dienes, 1996; Gasper, 1999; Isbell, 1999; Sinclair et al., 1994) suggesting that mood effects are largely due to the appraisal of the environment.

Several studies have shown that negative affect, such as anxiety and stress, can narrow the processing scope (Basso et al., 1996; B. L. Fredrickson \& Branigan, 2005; Karen Gasper \& Clore, 2002) and trigger an effortful, analytical processing style (Clark \& Isen, 1982; Isen, 1987). Studies on positive mood and cognition are also abundant and findings indicate that people in a good mood tend to reach decisions 
more quickly, use less information, and avoid analytical thinking. Happy people also appear to be more creative, open, and display greater cognitive flexibility (Ashby et al., 1999; Bless et al., 2006; Fiedler, 2001; Fredrickson, 2009; Hertel \& Fiedler, 1994; Isen \& Daubman, 1984). Other studies, however, have revealed that the reverse relationship between affect and cognitive style may also be true. Divergent thinking and global processing can make people happier than local processing (Akbari Chermahini \& Hommel, 2012; Ji et al., 2019). Therefore, the relationship between affect and processing style seem to be bi-directional and consistent. Positive affect should be associated with global holistic processing and an advantage in learning similarity-based categorization tasks. Negative affect should be associated with narrow local processing and an advantage in learning rule-based categorization tasks.

\section{Rationale of the Present Project}

The studies we have reviewed have generally examined the effects of developmental or temporary changes, but they have overlooked the possibility that executive attention, working memory capacity, and affect can also be studied as stable traits. Stable traits are often seen as biologically grounded tendencies that shape cognitive and behavioral processes. Studying traits would allow researchers to uncover more robust and long-lasting relationships. To my knowledge, there has only been one study that looked at the affective traits and processing style relationship (Basso et al., 1996), it found that trait-negative affect correlates with a local bias and trait-positive affect correlates with a global bias. However, more direct evidence is needed to clarify whether affective traits and the traits related to executive attention/working memory are associated with individual differences in category learning strategy use. In the present study, we examined the relationship between temperament traits and individuals' tendency to engage rule-based and similarity-based strategies, through learning a rule-based categorization task and a similarity-based categorization task.

\section{Temperamental Affect and Executive Attention}

Allport (1937, p. 54), a founder of the trait-based approach to personality, stated that "temperament refers to the characteristic phenomenon of an individual's emotional nature...dependent on constitutional make-up". The term 'constitutional' links temperament to underlying psychobiological systems and genetics. This definition is appealing because researchers can map temperament domains to underlying neurobiological systems to study their development and interaction. Rothbart and Derryberry (1979, 1981) similarly defined temperament as constitutionally based individual differences in emotional reactivity and self-regulation, and may form the basis of adult personality traits (Rothbart, 2012). Personality traits, on the other hand, extend to broader domains such as individuals' attitude and specific thought contents (Rothbart \& Bates, 2007). Temperament is relatively stable, but it may not fully stabilize until preschool years. This can be attributed to the brain's development of the executive attention system (e.g., lateral prefrontal cortex, and anterior cingulate cortex), which corresponds to the emergence of temperament trait of effortful control (Rothbart et al., 1994; Rueda, 2012) and coincides with children's ability to apply explicit rule-based strategies in category learning (Deng \& Sloutsky, 2016; Rabi \& Minda, 2014). Evans and Rothbart (2007) developed the Adult Temperament Questionnaire (ATQ) that measures four temperament factors: negative affect, extraversion/surgency, effortful control, and orienting sensitivity. Our study focused on the first three factors. The negative affect, extraversion/surgency and effortful control temperament factors map onto personality traits Neuroticism, Extraversion and Conscientiousness dimensions within the Big Five and Big Three scales of adult personality, respectively (Ahadi \& Rothbart, 1994; Evans \& Rothbart, 2007; Rothbart et al., 2000). 
The three temperament factors of interest are linked to the factors that influence an individual's learning strategy which we discussed in earlier sections. The negative affect and extraversion/surgency factors are related to emotional reactivity and refers to the tendency to experience negative and positive affect, respectively. The effortful control factor refers to the capacity to self-regulate, including attention control such as voluntarily focusing and shifting attention (Rothbart \& Rueda, 2005; Rothbart \& Bates, 2007). Posner and Rothbart have argued that the same brain regions (lateral prefrontal cortex, and anterior cingulate cortex) related to the executive attention network also support effortful control (Posner et al., 2007; Posner \& Rothbart, 2007). Whittle et al. (Whittle et al., 2008, 2009) also indicated that individual differences in effortful control are related to the structural and functional variations in the neural substrate corresponding to the executive attention network. From these, one can deduce that the results relating to executive attention can be used in making predictions for effortful control, and the findings of mood effects can provide grounds for making predictions for negative affect and extraversion/surgency. The present research extends from previous studies and examines the effects of stable fully-developed temperament traits on individual differences in the tendency to engage rule-based and similarity-based strategies on categorization tasks.

\section{General Hypotheses}

Following from the above discussion, we hypothesize that temperament traits should be predictive of individuals' category learning strategy. The dopaminergic theory contends that higher positive mood and lower negative mood leads to an increase in dopamine, which is associated with better verbal fluency in rule-based learning and better feedback processing in similarity-based learning. Therefore, the temperament profile - higher extraversion/surgency and lower negative affect should facilitate both rule-based and similarity-based category learning, and that this temperament advantage should be shown through faster discovery of task appropriate strategy and reaching a high level of performance.

The affect-as-information theory suggests that positive mood signifies a safe environment which encourages holistic and intuitive processing, whereas negative mood tends to elicit analytical processing in order to assess the problematic environment. The affect-as-information theory may be extended to predict affective traits effects. The temperament profile - higher extraversion/surgency and lower negative affect should be associated with a tendency to use similarity-based strategies and an advantage in learning similarity-based categorization tasks. The temperament profile - higher negative affect and lower extraversion/surgency should be associated with a tendency to use rule-based strategies and should show an advantage in learning rule-based categorization tasks.

Higher effortful control scores should be facilitative to learning either type of category structure, in terms of effectively applying task appropriate strategy. Other than self-rated scores of temperament factors, this study also measured objective working memory through a reading span task. It was hypothesized that higher working memory is beneficial to both types of category learning tasks in the same fashion as effortful control. 


\section{Methods}

\section{Materials}

Adult Temperament Questionnaire (ATQ; Evans \& Rothbart, 2007) includes 77 items which measure three temperament subscales from extremely untrue ( 1 out of 7 ) to extremely true ( 7 out of 7 ) on 7-point Likert scales. The subscales used in the current study were Negative Affect (e.g., It doesn't take very much to make me feel frustrated or irritated), Extraversion/Surgency (e.g., It doesn't take much to evoke a happy response in me), and Effortful control (e.g., I can keep performing a task even when I would rather not do it). It has been shown that ATQ subscales are closely related to factors within the Big Five adult personality framework. Specifically, Negative Affect, Extraversion/Surgency and Effortful Control map onto personality traits Neuroticism, Extraversion and Conscientiousness dimensions of the Big Five adult personality (Ahadi \& Rothbart, 1994; Evans \& Rothbart, 2007; Rothbart et al., 2000). This questionnaire was hosted on Western University's Qualtrics (https://mysurveys.uwo.ca/). At the end of the ATQ, the participants were given a clickable link which will redirect them to Pavlovia (pavlovia.org) to complete the next two tasks.

Reading Span Task (RSpan; Daneman \& Carpenter, 1980). This working memory task was originally designed by Daneman and Carpenter (1980). The current task was hosted on Pavlovia (Peirce, 2007), a platform that allows you to export locally-developed PsychoPy experiments online for easy data collection from many remote recruitment sources. On each trial, the participants read a sentence, they had to judge the meaningfulness of the sentence and also memorize the last word of the sentence for later serial recall. Each sentence appeared centrally on the screen, along with the text instruction -"Meaningful - press A", "Not Meaningful - press B" at bottom left and right, respectively. There were equal numbers of meaningful (e.g., "She knows many witty people") and meaningless (e.g., "Fish cut their nails after lunch") sentences. Participants had maximum 4.5 s to respond before the next trial began. They were informed that performance accuracy less than $80 \%$ on the meaningfulness judgment will not be included in the analysis. Although participants did not receive feedback on the meaningfulness judgment, their responses were recorded to assess potential sentence processing and memorization trade off (Robert et al., 2009). The trial sequence repeated two to six times depending on the set size. The intertrial interval was 500ms; and the order of set size was pseudorandomized, which means large set sizes can appear earlier than smaller set sizes. Following the set presentation, recall of the final words was prompted with "Please recall now" and a blank response box for typing. The participants typed as many words as they could recall from the current set. They were informed that the order of the words did not matter. There was no timing constraint for recall, participants clicked a radio-button after they finished typing. Feedback on recall was provided in the form of "You got X out of N correct!" (displayed for 1s).

There were altogether 60 sentences or 15 sets, the set size ranged from 2 to 6, each set size appeared three times. The sentences used in this task were selected from the sentence stimuli of Lewandowsky et al. (2010), sent through email correspondence from Dr. Klaus Oberauer. All of the sentences contained between 3 and 6 words. All final words contained between 3 and 6 letters, and had 1 or 2 syllables. Practice trials were provided before the actual task, the participants first learned the meaningfulness judgment part through four trials, then learned the memorization part through another four trials, and finally they practiced one set of size 4 that followed the procedures of the actual task. Participants continued to the category learning task after completing the reading span task within the same PsychoPy experiment. 
Category Learning Task (an adaptation to Le Pelley et al., 2019). The category stimuli used in the present study were lines varying in length and angle. This category learning task was built into the same online experiment link as RSpan task on Pavlovia, the participants were presented with this task upon finishing the RSpan task. Each stimulus was a single blue line presented within a white square with gray borders. Table 1 shows the parameters that were used to generate the stimuli for the Conjunction Rule-based (CR) and Information Integration (II) categories, and Fig. 1 shows the resulting category structures. On each trial, the participants saw a line, along with the question "Does this line belong to Category A or Category B?", to which they made a decision by pressing A or B on the keyboard. There was no timing constraint for responding. Feedback was provided immediately after the keyboard response. The participant was either shown the word "Correct" in green or "Wrong" in red (presented centrally for $600 \mathrm{~ms}$ ), followed by a blank interval of $200 \mathrm{~ms}$ before the next trial began. Each participant completed 12 sets of 30 trials each (360 trials total), they had the option to take a self-paced break after each set of 30 trials. The CR task and II task had identical experimental setup. The reason that the categories within the II tasks are further away from the decision bound and formed tighter clusters than categories within the $\mathrm{CR}$ task is because these specific parameters encouraged above-chance performance. Our piloting data suggests that when similar stimuli spreadedness were used for the II task, it was more difficult to learn than the CR task.

Table 1. Parameters for stimulus generation in the conjunction and information integration conditions.

\begin{tabular}{|l|l|l|}
\hline & \multicolumn{2}{|l|}{ Parameter } \\
\hline Category & $\boldsymbol{\mu}$ length & $\boldsymbol{\mu}$ angle \\
\hline Conjunction & & \\
\hline A & 100 & 200 \\
\hline B & 100 & 100 \\
\hline B & 200 & 100 \\
\hline B & 200 & 200 \\
\hline Information Integration & & \\
\hline A & 80 & 150 \\
\hline A & 150 & 220 \\
\hline B & 150 & 80 \\
\hline B & 220 & 150 \\
\hline
\end{tabular}

Note: $\boldsymbol{\mu l e n g t h}$ and $\boldsymbol{\mu}$ angleare the means of the normal distributions (CR: $\sigma_{\text {length }}=10$ and $\sigma$ orientation $=900 ; \mathrm{II}: \sigma_{\text {length }}=30$ and $\sigma_{\text {orientation }}=500$ ) that were sampled to generate the length and angle (respectively) of the stimuli in the categorization task. For length, the given value was divided by 500 when presented on Pavlovia to give the length of the line stimulus in pixels. For angle, the given value was multiplied by $\pi / 500$ to give the angle of the line from horizontal in radians. The order of stimuli presentation was pseudorandomized, all 
participants of a given category structure received the exact same set of line stimuli in the same order. Half of the stimuli had Category A as the correct answer, and half had Category $\mathrm{B}$ as the correct answer.
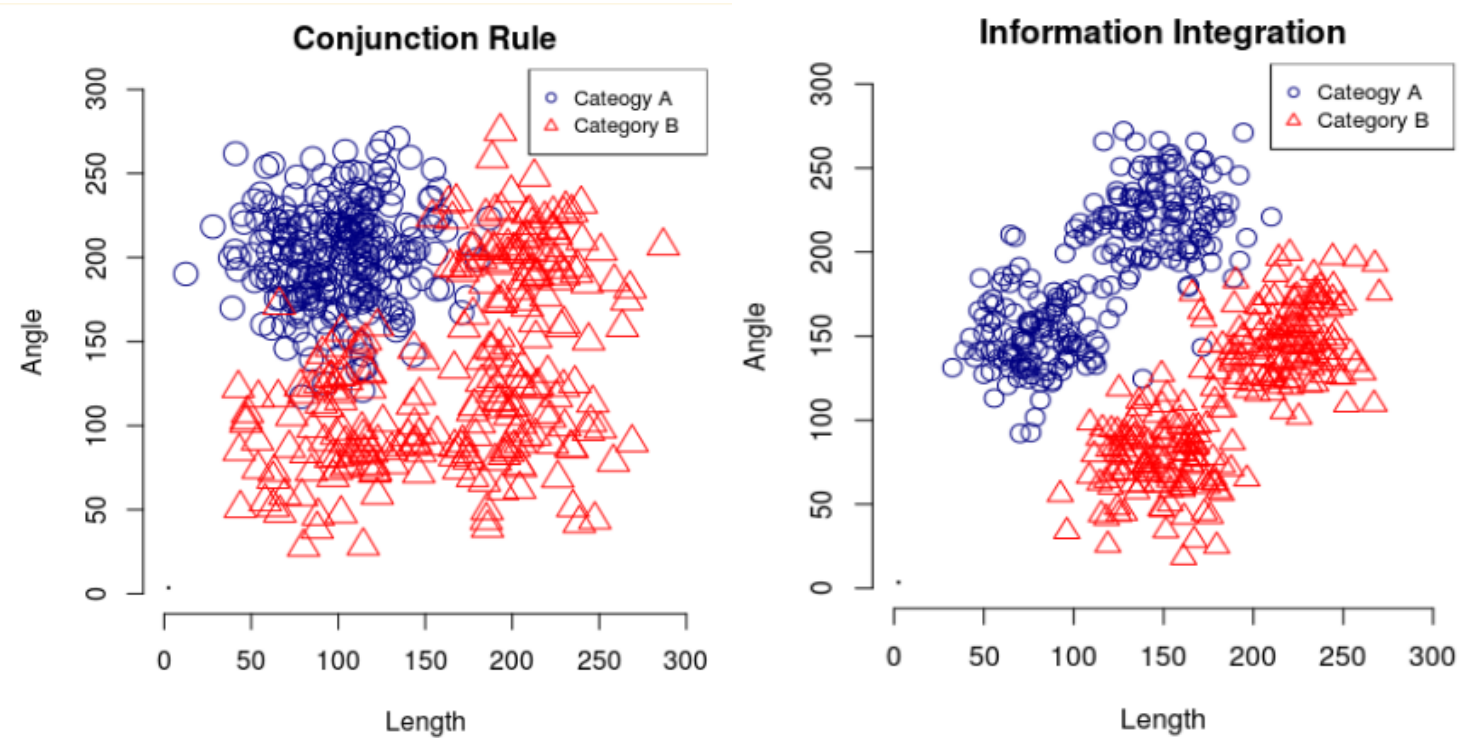

Figure 1. The conjunction and information integration category structures used in the current experiment. Values on each dimension refer to the length and angle of the line stimuli in the categorization task. Values are in arbitrary units.

\section{Procedure}

Participants signed up through either Western's participant pool or Amazon MTurk. They clicked the provided link to access the ATQ which was hosted on Western's Qualtrics server. At the end of the questionnaire, participants were given another link that re-directed them to Pavlovia--where the RSpan and category learning tasks were hosted. The entire process took on average 35 minutes, participants were compensated either 0.5 course credit or $\$ 2$ for their time.

\section{Participants and Descriptive Statistics}

CR Task. Two-hundred and eighty-four participants were recruited. All of them completed the ATQ on Qualtrics, but only 196 followed the instruction to complete the entire experiment (reading span and categorization task). Since the data for the two parts of the experiment were stored separately (Qualtrics and Pavlovia), each participant's data was linked by an unique ID which they were asked to enter in both parts of the experiment. However, among the 88 participants whose data was unusable, most have failed to enter an ID or entered a different ID on each part, which resulted in us unable to link the two parts for analyses. A smaller proportion of incomplete data was resulted from participants ending the experiment prematurely out of their own free will or browser issue when clicking the link to the second part.

Of the 196 participants (17 from MTurk, the rest of the participants were undergraduate students from Western University) who completed the Conjunction Rule (CR) categorization task, along with ATQ and reading span (Age: $M=21.56$, $s d=9.72$ ). See Table 2 for more participants' demographic information. 
The majority of participants were from Canada $(\mathrm{N}=123)$, 19 were from the U.S (all were MTurk participants), the remaining participants were from a variety of countries and were studying in Canada. Eight participants indicated they had a mental or physical impairment.

Table 2. Demographic information of participants in the CR task.

\begin{tabular}{|c|c|}
\hline Gender & \\
\hline Male & 44 \\
\hline Female & 152 \\
\hline \multicolumn{2}{|l|}{ Ethnicity } \\
\hline Asian/Pacific Islander & 46 \\
\hline Black/African Canadian & 7 \\
\hline Hispanic/Latino & 4 \\
\hline Multiracial/Biracial & 7 \\
\hline Native Canadian & 1 \\
\hline South Asian & 30 \\
\hline White/Caucasian & 85 \\
\hline Not listed & 16 \\
\hline \multicolumn{2}{|l|}{ Highest Education Achieved } \\
\hline Below high school & 2 \\
\hline High school & 170 \\
\hline Bachelor's degree & 15 \\
\hline Master's degree & 4 \\
\hline College/vocational school & 5 \\
\hline
\end{tabular}

Temperament was measured through participants' self-report on the Adult Temperament Questionnaire (ATQ). The three temperament factors of interest were: negative affect, extraversion/surgency and effortful control. The final numerical score of each factor for each participant was calculated by adding up the ratings on all items associated with this factor, then dividing the total number of items. The scores for factor negative affect ranged from 2.52 to 5.88 (Median $=4.42, M=4.44$, $s d$ $=0.67$ ); the scores for factor extraversion/surgency ranged from 2.24 to 6.35 (Median $=4.77, M=4.68$, sd = 0.83 ); the scores for factor effortful control ranged from 2.00 to 5.74 (Median $=3.95, M=3.95$, $s d=0.79$ ). Participants were divided into higher or lower groups on the first two temperament factors based on a median split (negative affect: high $n=99$, low $n=97$; extraversion/surgency: high $n=105$, low $n=91$ ). Two temperament profiles were then extracted based on the high/low grouping of negative affect and 
extraversion/surgency: high negative affect, low extraversion/surgency (highN/lowEx; $\mathrm{n}=57$ ); low negative affect, high extraversion/surgency (lowN/higherEx; $\mathrm{n}=63$ ). These profiles were of particular interest because only one of the affective factors was high. We would be able to examine the effect of either negative affect or extraversion/surgency without the other exerting an effect in the opposite direction.

II Task. Two-hundred and forty-five participants were recruited. Forty-seven participants either did not enter the same unique ID on both parts of the experiment, terminated the experiment prematurely, or experienced browser issues upon clicking the link. That left 198 participants (7 from MTurk, the rest of the participants were undergraduate students from Western University) who followed the instruction strictly and completed the Information Integration (II) categorization task, along with the temperament questionnaire and reading span task (Age: $M=18.90, s d=1.26$ ). See Table 3 for more participants' demographic information. The majority of participants were from Canada $(\mathrm{N}=141), 18$ were from China, the remaining participants were from a variety of countries and were studying in Canada. Nine participants indicated they had a mental or physical impairment.

Table 3. Demographic information of participants in the II task.

\begin{tabular}{|c|c|}
\hline Gender & \\
\hline Male & 64 \\
\hline Female & 134 \\
\hline \multicolumn{2}{|l|}{ Ethnicity } \\
\hline Asian/Pacific Islander & 41 \\
\hline Black/African Canadian & 3 \\
\hline Hispanic/Latino & 2 \\
\hline Multiracial/Biracial & 12 \\
\hline South Asian & 40 \\
\hline White/Caucasian & 91 \\
\hline Not listed & 9 \\
\hline \multicolumn{2}{|l|}{ Highest Education Achieved } \\
\hline High school & 190 \\
\hline Bachelor's degree & 6 \\
\hline College/vocational school & 2 \\
\hline
\end{tabular}

The scores for factor negative affect ranged from 2.40 to 6.52 (Median $=4.48, M=4.49$, sd = 0.64); the scores for factor extraversion/surgency ranged from 2.35 to 6.47 (Median $=4.71, M=4.69$, sd = 0.73 ); the scores for factor effortful control ranged from 1.68 to 5.37 (Median $=3.79, M=3.77$, sd $=0.67$ ). Participants were 
divided into higher or lower groups on the first two temperament factors based on a median split (negative affect: high $\mathrm{n}=97$, low $\mathrm{n}=101$; extraversion/surgency: high $\mathrm{n}=97$, low $\mathrm{n}=101$ ). Two temperament profiles were then extracted based on the high/low grouping of negative affect and extraversion/surgency scores: high negative affect, low extraversion/surgency, (highN/lowEx; $\mathrm{n}=61$ ); low negative affect, high extraversion/surgency(lowN/highEx; $\mathrm{n}=61$ ).

\section{Data Analysis and Formal Modeling}

\section{Behavioural Data Analysis}

The CR and II categorization task each had 12 blocks of 30 trials. In the analysis, 2 subsequent blocks were combined into one larger block to both aid later model-based analysis and be comparable to other studies that have used similar tasks. This produced six 60-trial blocks. All mentions of 'block' from now will be referring to these 60 -trial blocks.

The primary dependent measure was the proportion of correct responses in the first and last category learning block. First, we conducted Welch Two-Sample t-tests to examine the affective temperament effects on proportion correct in the first and last block of the CR and II task. These analyses were done after removing the participants who had less than $60 \%$ overall performance in learning the category structure they were assigned to.

Next, linear regression analyses were carried out to complement t-tests results, effortful control (measure of executive attention) and number of words recalled from RSpan (measure of working memory storage) were included as predictors in the models. The inclusion of the RSpan was intended to scrutinize the robustness of the affective temperament effects by having a previously-shown significant predictor of category learning to partial out a certain amount of variances in the dependent variable. For the regression analyses, participants who had less than $80 \%$ accuracy on the meaningfulness judgment part of the RSpan task were also removed. Meaningfulness judgment was a measure of sentence processing, low performance on this part may imply a tradeoff between processing and memorization, which may render the number of words recalled less good an indicator of working memory.

Moreover, despite that the evidence around the dopaminergic theory has been less consistent, it would still be interesting to see whether this theory of transient affect can be useful in making predictions for trait affect. Multiple regression analysis was conducted for each of the category learning tasks, with the first optimal strategy block number as the dependent variable. Participants who never used the task appropriate strategy were given a ' 7 ' on this measure (there were only 6 blocks in the tasks). These analyses should tell us whether the two temperament profiles differed in their default strategy and speed of optimal strategy discovery.

\section{Decision Bound Computational Modeling}

We also modeled participants' category responses in the first and last block to investigate the categorization strategy they started with and eventually transitioned into. Moreover, modelling analysis was used to examine whether each temperament profile was biased towards one strategy over the others. We fitted five different models to the first and final block of each participant's data using the grt package (Matsuki, 2014) in the R environment. The models differed in terms of the hypothesized boundaries that best divide the two categories in the category space shown in Fig. 1. The models used in this analysis were as follows: 
The unidimensional models assume that the participants divide the two categories along one of the stimulus dimensions, either orientation or length. The unidimensional models we used had two parameters: the intercept value on the stimulus dimension of interest, and noise.

The conjunction model assumes that the participants incorporate both stimulus dimensional information to form a rule, which they then use to divide the two categories. Specifically, participants evaluate each dimension separately first, then combine these evaluations to form a categorization decision. For instance, a conjunction rule can be "Assign to Category A if the line is short and the top is tilted to the left, otherwise assigned to Category B." The conjunction model we used had three parameters: the intercept value on each of the stimulus dimensions, and noise. This is the optimal strategy for learning the CR categorization task, we refer to this model as the CR strategy in the rest of the paper.

The diagonal model assumes that the best category-divider is a diagonal line. The stimulus dimensions need to be integrated prior to making a categorization judgment. The diagonal model has three parameters: the intercept and slope of the decision bound, and noise. This is the optimal strategy for learning the II categorization task, we refer to this model as the II strategy in the rest of the paper.

The random guessing models assume that there is no pattern behind participants' responses, this model has no parameters.

Maximum-likelihood parameter estimation was used by the grt package to determine the intercept (and slope) for each model that can best divide each participant's A and B responses. Next, we used the Bayesian information criterion (BIC: Schwarz, 1978) to select the best-fitting model for the two blocks of each participant.

\section{Results}

\section{Early and Late Categorization Accuracy}

CR task. Seventeen participants were removed because of less than $60 \%$ overall performance. This procedure left 57 participants with highN/lowEx temperament profile and 54 participants with lowN/highEx temperament profile in the analyses. Accuracy on the CR task can be found in Table 2. Table 3 presents the best block performance for each temperament profile. Welch Two-Sample t-tests were conducted to compare the temperament profiles' overall categorization accuracy, and the accuracy in the first block and the last block. Overall accuracy did not differ between temperament profiles, $t(109)=-$ $0.31, p=.76$. First block performance did not differ between temperament profiles, $t(109)=1.41, p=.16$, neither did performance in the last block, $t(109)=-0.38, p=.70$ (see Fig. 2 left). These results suggest that participants performed comparable throughout the CR task, regardless of their temperament profile.

II task. Twenty-three participants were removed because of less than $60 \%$ overall performance. This procedure left 54 participants with highN/lowEx temperament profile and 54 participants with lowN/highEx temperament profile in the analyses. Accuracy and best performing block information can be found in Table 4 and Table 5. Overall accuracy did not differ between temperament profiles, $t(106)=$ $0.69, p=.49$. First block performance did not differ between temperament profiles, $t(106)=1.31, p=.19$. In the last block, the performance difference between temperament profile lowN/highEx and highN/lowEx approached significance, $t(106)=-1.81, p=.073$, the lowN/highEx profile $(M=85.06)$ participants performed better than highN/lowEx profile $(M=81.34)$ participants (see Fig. 2 right). These results suggest that participants with different temperament profiles performed similarly at the beginning of learning, but the ones with the lowN/highEx profile continued to improve until the end of the II 
categorization task and may have achieved better performance than participants with the highN/lowEx profile.

Table 4. Mean category learning performance on the CR and II task.

\begin{tabular}{|c|c|c|c|}
\hline & All participants & highN/lowEx & lowN/highEx \\
\hline CR task & $71.20(8.68)$ & $73.05(6.73)$ & $73.46(6.24)$ \\
\hline II task & $77.97(11.74)$ & $82.27(7.01)$ & $81.72(7.54)$ \\
\hline
\end{tabular}

Note. The 'All participants' column shows the accuracy of all participants who completed the CR or II task regardless of their temperament composition. SD are listed in parentheses. highN/lowEx represents the participants with high negative affect and low extraversion temperament profile; lowN/highEx represents the participants with low negative affect and high extraversion temperament profile.

Table 5. Best block performance on the CR and II task for each temperament profile.

\begin{tabular}{|l|l|l|}
\hline & highN/lowEx & lowN/highEx \\
\hline CR task & Block 3 - 78.24 (9.00) & Block 5 - 76.99 (9.75) \\
\hline II task & Block 3 - 85.15 (9.13) & Block 6 - 86.01 (8.62) \\
\hline
\end{tabular}
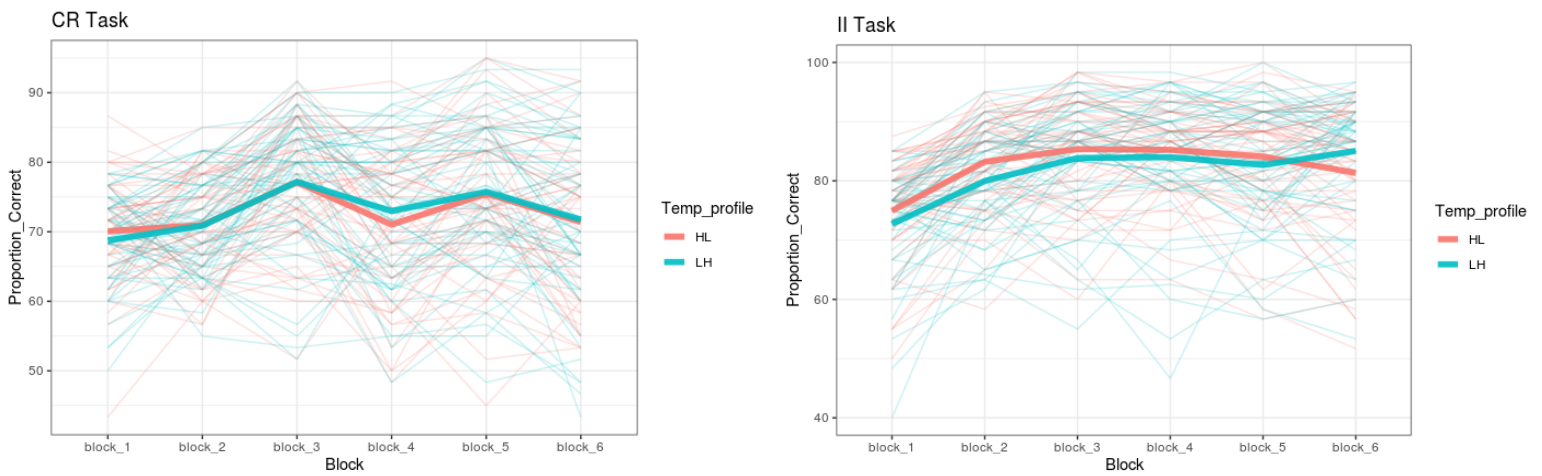

Figure 2. Categorization accuracy across the six blocks for each temperament profile. The dark, solid lines denote the average accuracy for each temperament profile. The lighter lines represent each individual participant's performance. Categorization accuracy is defined as the proportion of correct trials per block. Left figure shows performance on the CR task; the right figure shows performance on the II task. highN/lowEx = high Negative Affect, low Extraversion/surgency; lowN/highEx = low Negative Affect, high Extraversion/surgency 


\section{Effect of effortful control and Working Memory}

The temperament factor effortful control was hypothesized to aid with effective categorization learning. Moreover, previous studies have suggested that working memory may differentially affect people's categorization performance and strategy use.

CR Task. An additional 8 participants were removed because of lower than $80 \%$ accuracy on the meaningfulness judgment part of the RSpan task. After this procedure, the highN/lowEx profile had 53 participants and the lowN/highEx profile had 52 participants. A multiple regression analysis was conducted with the first block performance as the dependent measure; temperament profile, effortful control and working memory were independent variables. The Backwards elimination process using the "step" function from the "stats" package in R removed all predictors from the model ((R Core Team, 2020). None of the predictors significantly predicted the dependent variable [intercept: $\beta=69.17, S E=$ $0.69, t(102)=99.75, p<.001]$. Another multiple regression analysis was conducted with temperament profile, effortful control and working memory regressed on the final block categorization performance. The backwards elimination process removed temperament profile and working memory from the model. The remaining predictor effortful control was only approaching significance, $[\beta=2.56, S E=1.41, t(102)=$ $1.82, p=.072]$. This showed that temperament profile, effortful control and performance on the RSpan were not significantly related to $\mathrm{CR}$ task performance.

II Task. An additional 19 participants were removed because of lower than $80 \%$ accuracy on the meaningfulness judgment part of the RSpan task. After this procedure, the highN/lowEx profile had 45 participants and the lowN/highEx profile had 48 participants. A multiple regression analysis was conducted with the first block performance as the dependent measure; temperament profile, effortful control and working memory were independent variables. The Backwards elimination process removed temperament profile and effortful control as predictors from the model. The effect of working memory was significant, $[\beta=0.29, S E=0.09, t(91)=3.19, p=.002]$, participants with higher working memory had higher categorization accuracy than participants with lower working memory. These results suggest that participants' performance on the first category learning block relied significantly on their working memory, and not on their affective temperament profile or effortful control.

Another multiple regression analysis was conducted with temperament profile, effortful control and working memory regressed on the final block categorization performance. The Backwards elimination process removed effortful control as a predictor from the model. In the reduced model, the effect of temperament profile was significant, $[\beta=5.59, S E=2.06, t(90)=2.71, p=.008]$, participants with the lowN/highEx temperament profile performed better than participants with highN/lowEx temperament profile. The effect of working memory was significant, $[\beta=0.23, S E=0.11, t(90)=2.13, p=.036]$, participants with higher working memory performed significantly better than participants with lower working memory. These results suggest that despite the effortful control score being different between the temperament profiles, it was not predictive of the last block's performance. Furthermore, even though working memory was a significant predictor of categorization performance, it was not significantly different between the two temperament profiles. Therefore, the underlying affective temperament was the intrinsic reason behind final categorization learning performance difference.

\section{Model-based Analysis}

Participants' estimated strategy use was compared between the two temperament profiles in both early and late learning to examine whether the temperament profiles are predictive of strategy use. This 
analysis also shows whether the difference in performance accuracy described in the earlier section was related to strategy selection.

CR Task. Fisher's exact test was used to examine whether the number of participants who were fit best by each strategy model differ across temperament profiles in early and late learning. There were no differences in strategy use among the two temperament profiles in early learning (Fisher's Exact Test, $p=$ .37) or late late learning $(p=.88)$. Figure 5 shows the strategy distribution across participants within the two temperament profiles. It is worth noting that the majority of participants used a single dimensional rule in the first block as suggested by previous literature; but contradicting past studies, the largest strategy group in the last block used information integration approach rather than the optimal conjunction rule ${ }^{1}$. It was unexpected that a large and comparable proportion of participants from both temperament profiles settled with $75 \%$ accuracy or less. The results showed that the two temperament profiles did not differ in their strategy use in either the early or late stage of learning the CR categorization task.

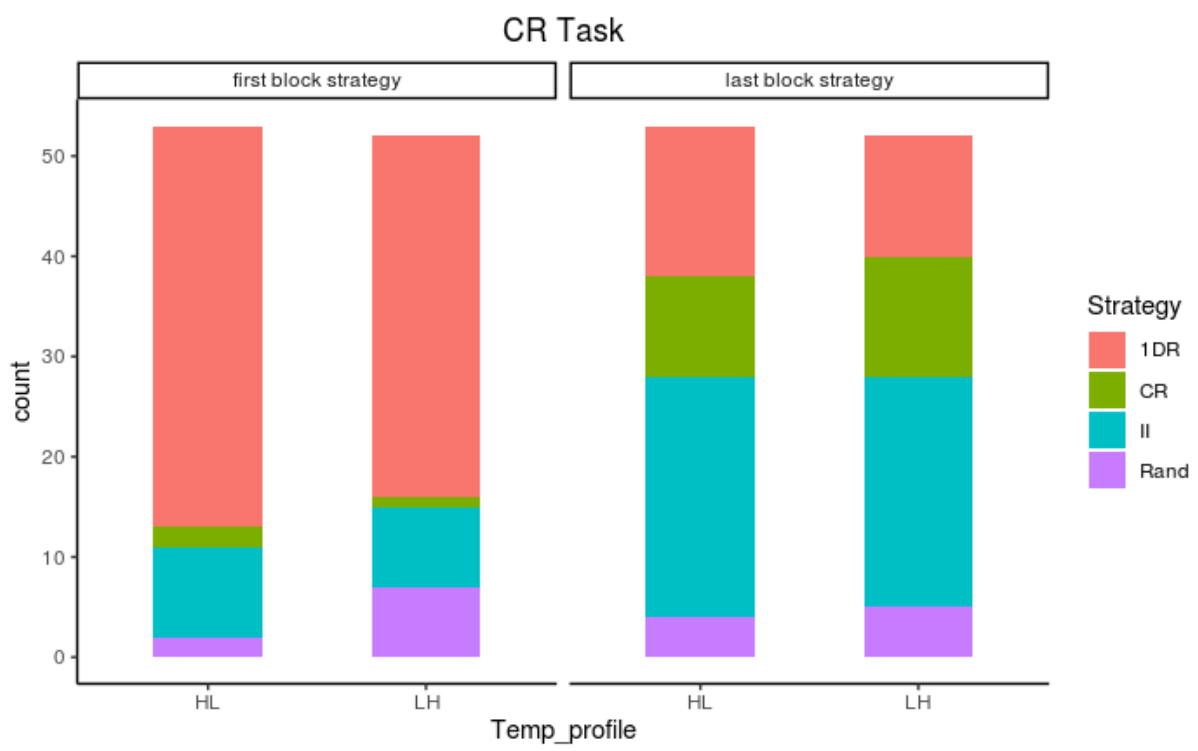

Figure 5. The number of participants who were fit best by each strategy model among the two temperament profiles in the first and last category learning block on the CR task. Strategy usage did not differ in the first or last block between the two temperament profiles. highN/lowEx $=$ high Negative Affect, low Extraversion/surgency; lowN/highEx = low Negative Affect, high Extraversion/surgency; 1DR = single-dimensional

\footnotetext{
${ }^{1}$ A one-way ANOVA was conducted to compare the final block accuracy between the strategy groups (CR: $M=$ 84.17; II: $M=74.93$; 1DR: $M=63.02$; Rand: $M=55.19)$. There was a significant effect of strategy on performance at $p$ $<.001$ level for the four types of strategies, $[F(3,101)=51.02, p<.001]$. Post hoc comparisons using the Tukey HSD test indicated that CR strategy was associated with significantly better performance than all of the other strategies at $p<$ .001 level; participants who used II strategy performed significantly better than people who used unidimensional rule or random guessing at $p<.001$ level; the performance difference between unidimensional rule and random guessing was marginally significant, with unidimensional strategy being associated with better performance at $\mathrm{p}<.05$ level.
} 
rule strategy; $\mathrm{CR}=$ conjunction rule strategy; II = information-integration strategy; Rand = inconsistent/random responder

II Task. Fisher's exact test was used to examine whether the number of participants who were fit best by each strategy model differ across temperament profiles in early and late learning. There were no differences in strategy use among the two temperament profiles in early learning of the II task $(p=.46)$, but the difference was significant between highN/lowEx and lowN/highEx profiles at the end of learning $(p=.015 \text {; Fig. } 6)^{2}$. Figure 6 shows the strategy distribution across participants within the two temperament profiles. Further inspection showed that participants with the temperament profile lowN/highEx were more likely to use the optimal II strategy by the end of the II task than participants with the highN/lowEx profile ( $n=40$ vs. $n=29$ ). Strategy analysis showed that all participants started in the same place in terms of strategy usage, but participants with the lowN/highEx temperament profile were able to better transition into using the optimal II strategy than the highN/lowEx profile by the end of the II categorization task. These results suggest that the higher accuracy seen in late learning in the lowN/highEx temperament group was indeed due to more usage of the optimal II strategy.

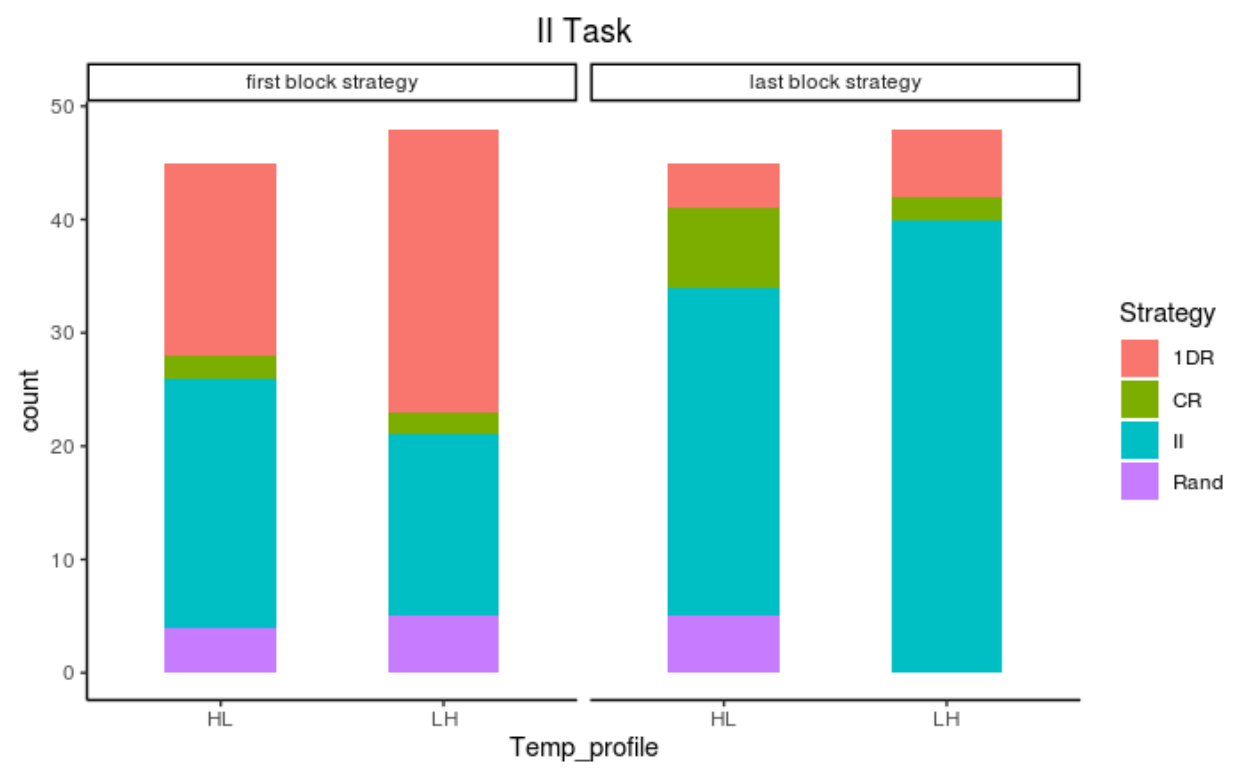

Figure 6. Distribution of strategy usage among the two temperament profiles in the first and last category learning block on the II task. Strategy usage differed significantly in the last block between the two temperament profiles. highN/lowEx = high Negative Affect, low Extraversion/surgency; lowN/highEx = low Negative Affect, high Extraversion/surgency; $1 \mathrm{DR}=$ single-dimensional rule strategy; $\mathrm{CR}=$ conjunction rule strategy; II = information-integration strategy; Rand = inconsistent/random responder

\footnotetext{
${ }^{2}$ A one-way ANOVA was conducted to compare the final block accuracy between the strategy groups (CR: $M=$ 83.33; II: $M=87.67$; 1DR: $M=68.17$; Rand: $M=58.33)$. There was a significant effect of strategy on performance at $p$ $<.001$ level for the four types of strategies, $[F(3,89)=65.62, p<.001]$. Post hoc comparisons using the Tukey HSD test indicated that participants who used II strategy did not perform significantly better than people who used CR strategy, ns; both CR and II strategy was associated with significantly better performance than unidimensional rule or random guessing at $p<.001$ level; the performance difference between unidimensional rule and random guessing was significant, with unidimensional strategy being associated with better performance at $\mathrm{p}<.05$ level.
} 


\section{Speed of Optimal Strategy Discovery}

Two multiple regression analyses were conducted, one for the CR task and one for the II task, to examine whether affective temperament traits are associated with task appropriate strategy discovery speed, effortful control and working memory were also included in the models. The Backwards elimination process removed all three predictors in both regression models. These results indicated that the two temperament profiles did not differ in their default strategy use and it took a comparable amount of learning for these two groups to discover the optimal strategy, regardless of the strategy being rule-based or similarity-based.

\section{Discussion}

\section{Summary of Results}

The goal of the current study was to examine whether temperament traits can predict people's tendency to use rule-based and/or similarity-based strategies in category learning tasks. Results showed that participants with the lowN/highEx temperament profile had an advantage in learning the II categorization task over participants with the highN/lowEx profile, this was shown through higher performance accuracy and more optimal II strategy use in the final block. These results supported the predictions made by both the dopaminergic theory and the affect-as-information hypothesis. The dopaminergic theory suggests that the increase in dopamine due to higher positive affect/lower negative affect facilitates feedback processing in similarity-based learning; whereas the affect-as-information hypothesis contends that individuals perceive the environment as safe when they are in a positive mood and thus rely on intuitive, similarity-based processes. There were no differences between the two temperament profiles for participants learning the CR categorization task. Results neither supported the affect-as-information hypothesis that highN/lowEx profile should be associated with more rule use and better performance, nor did they support the dopaminergic theory that low $\mathrm{N} /$ highEx profile should be associated with more optimal strategy use and better performance. Contrary to the predictions, self-rated executive attention (effortful control) was not a significant predictor of performance on either task in any learning stage. Interestingly, working memory storage significantly predicted people's early and final stage performance accuracy on the II task, but it was not a predictor on the CR task. Moreover, the two temperament profiles did not differ in their default strategy, it took a comparable amount of learning for the two groups to discover the optimal strategy in both the CR and the II tasks.

\section{Affective Temperament Effects on Rule-based vs. Similarity-based Learning}

Ashby et al. (1998) proposed the dopaminergic theory of positive affect within the cognitive psychology literature. It argues that the effects of positive affect on cognition are mediated by dopamine, and increase of dopamine release in the anterior cingulate during transient positive mood states increases cognitive flexibility and verbal fluency. Ashby and colleagues suggest that positive affect facilitates both explicit rule-based learning and implicit similarity-based learning. Within social psychology literature, the affect-as-information hypothesis (Clore, 1992; Schwarz \& Clore, 1983) proposes that people rely on their mood to judge the nature of the environment. Positive affect signifies that the environment is safe, therefore people tend to engage in heuristic and holistic processing; if the negative mood was prominent, then people would switch to a more analytical processing style to assess the problematic environment.

Affect has often been studied as short-lived experiences, to my knowledge, the current study is one of the first that explored the relationship between temperament traits of affect and the tendency to use 
rule-based or similarity-based approaches. Affects are basic components of a person's temperament (Goldsmith et al., 1987). (Allport, 1937, p. 54) linked temperament to underlying psychobiological systems and genetics. The current study used temperamental traits positive and negative affect to explore a more lasting relationship between affect and processing bias that goes beyond transient moods. Two temperament profiles were created, the first profile was composed of individuals with lower negative affect and higher extraversion/surgency and the second profile was composed of people with higher negative affect and lower extraversion. These two profiles represented the extremes of affective temperament traits and are most likely to show a difference in strategy use if there was indeed a difference.

Interpretation of CR task results. Affective temperament traits did not predict participants' tendency to use rule-based or similarity-based strategy on the CR task. Contrary to the predictions of dopaminergic theory, the low $\mathrm{N} /$ highEx profile did not show a performance advantage, and contrary to the predictions of affect-as-information hypothesis, the highN/lowEx profile did not perform better either. Results from the CR task suggest that theories of transient mood effects cannot be directly used to make predictions for affective traits. First, affective traits influence cognition gradually and may not be as salient as experimentally manipulated mood states. Moreover, the present study did not measure participants' current mood states at the time of their participation, it is possible that affect states have interacted with affective traits and led to the null results. Although this is unlikely since we had a large sample size, the variations in current mood states should have been evened out. Second, feedback was provided in the present study, which may have swayed participants' strategy towards task appropriate and away from the default. The supervised learning environment is more stringent in measuring strategy use tendency compared to tasks that allow participants to freely respond without accuracy feedback (Basso et al., 1996; McDaniel et al., 2014; Murphy et al., 2017). We relied on feedback-driven methods in order to facilitate learning and discourage arbitrary responses. Third, prior research has shown that the effects of emotion were not consistently found through emotional-neutral tasks (Derryberry \& Reed, 1994; Easterbrook, 1959; Isen, 1970). Given the emotion-neutral nature of the categorization task in this study, it can be speculated that no effects have been elicited by affective temperament traits. These speculations are based on the assumption that affective traits have the same effects on cognition as transient emotions, but just milder. There could also be the possibility that affective traits have cumulative effects on completely different neurological networks than the ones involved in transient emotions, future studies may want to explore more into this possibility.

In our opinion, the results from the $\mathrm{CR}$ category learning task did not necessarily refute previous theories or findings of transient mood effects, rather, they extended the literature of emotion and cognition. This study provides empirical data for the relationship between affective temperament traits and rule-based category learning.

Interpretation of II task results. Affective temperament traits predicted participants' tendency to use rule-based or similarity-based strategy on the II task. The lowN/highEx profile was associated with more task appropriate strategy use and better performance than the highN/lowEx profile in the final learning block.

The dopaminergic theory postulates that positive affect facilitates similarity-based learning through projecting dopamine into the striatum--a critical region for this type of learning (Ashby et al., 2007). Moreover, positive affect improves cognitive flexibility, which in turn can speed up the process of exhausting potential rule-based strategy before resorting to the similarity-based strategy. Therefore we should expect to see the lowN/highEx profile learn the task appropriate strategy faster and reach high performance sooner than the highN/lowEx profile. Figure 2 shows that the two temperament profiles 
performed similarly throughout the first 5 learning blocks, and they required a comparable amount of learning to discover the optimal strategy. Therefore, the dopaminergic theory cannot fully explain the observed data. The affect-as-information hypothesis (Clore, 1992; Schwarz, 1990) proposes that people rely on affect to judge whether the environment is safe, positive affect signifies a safe environment, thus people tend to engage in holistic and heuristic processing. Much of the evidence for the affect-as-information hypothesis comes from free response tasks which measure participants' default tendency to focus on local or global features (Bodenhausen et al., 1994; Dienes, 1996; Wyer et al., 1999). This study did not find a difference in processing being reflected through strategy use in the early stage of learning. Even though strategy use difference was detected in later learning, the affect-as-information hypothesis is not able to account for the full picture results.

Alternative accounts may provide some insights in light of the results. The task used in this study was long and repetitive (the categorization task took at least 20 minutes to complete), which could have created a frustrating situation for the participants. A follow-up analysis that compared the frustration subscale self-rated scores (a subscale within the negative affect factor) across the two temperament profiles had shown that the highN/lowEx profile self-rated significantly higher on being easily annoyed and irritated than the low $\mathrm{N} /$ highEx profile ${ }^{3}$. Not surprisingly, participants with the highN/lowEx profile were in a much more agitated state than the lowN/highEx profile participants during the task. Frustration has been categorized as a type of aggressive negative affect with strong motivational intensity (Evans \& Rothbart, 2007). Gable and Harmon-Jones (2008; 2010) suggested that affective states that are high in motivational intensity tend to narrow attention. It can be speculated that participants with the highN/lowEx profile experienced heightened levels of frustration thus started using task inappropriate rule-based strategies in the final block. This speculation is under the assumption that affective traits can help determine the likelihood of an individual experiencing positive or negative affective states in a certain situation. Moreover, Folkman and Moskowitz (2000) linked the occurrence and maintenance of positive affect to giving ordinary events positive meaning and positive reappraisal of negative events. Therefore, people with the lowN/highEx temperament profile not only were less frustrated they also may have used problem-focused positive reappraisal to continue to improve their categorization performance. Therefore, different levels of frustration and different appraisal of negative situations associated with the temperament profiles may have led to the performance and strategy use differences between the two profiles in the final block.

\section{Effects of Executive Attention and Working Memory}

Effects of Executive Attention. The definition of temperament has often been related to emotional reactivity (Allport, 1937; Pickford et al., 1954), but executive attention is also an important component (Rothbart et al., 1994). Executive attention was measured in this study through the effortful control factor within the ATQ. Rueda (2012) describes effortful control as a voluntary mode of control that provides the individual flexible amount of resources to respond to the situation, and this control is linked to the executive attention network (Posner et al., 2007; Posner \& Rothbart, 2007). In both the CR and II category learning tasks, the two temperament profiles differed significantly in their self-reported effortful control scores, with the lowN/highEx profile rated higher than the highN/lowEx profile. Prior studies have suggested that higher executive attention is associated with better learning on both rule-based and similarity-based categorization tasks (Maddox et al., 2010; Rabi \& Minda, 2016). Other studies found that individual differences in executive attention was only related to learning complex rules, but not simple

${ }^{3}$ ATQ frustration subscale scores differed significantly between the two temperament profiles, $t(91)=5.25, p<.001$, with the highN/lowEx profile self-rated higher than the lowN/highEx profile. 
rules or similarity-based strategy (Minda et al., 2008; Rabi \& Minda, 2016; Racine et al., 2006). However, this study found that executive attention did not independently predict category learning performance in either CR or II tasks. Moreover, none of the tested predictors were significant in the CR task in both early and late learning, and most participants have settled with a strategy that yielded performance less than $75 \%$. This may suggest that this specific version of the CR task was too difficult to learn for most individuals regardless of the levels of their temperament traits and working memory. Another potential explanation for the low performance among all participants of the CR task may be high stress level. Undergraduate students were stressed at many levels because of school work and the pandemic, this stress may constantly drive their attention away from task at hand and towards stress-related subjects (Eysenck et al., 2007). It may be the case that a large proportion of participants were not able to reach their full learning potential under constant stress, which may have blurred the potentially significant effects of the predictors. The reason why this pattern was only seen in the CR task but not the II task may be that the CR strategy requires effortful analytical processing whereas the II strategy can be applied more or less automatically, thus CR task performance may be more strongly impacted by divided attention. In addition, there could be many distractions in a participant's environment, such as noise or web pages in the background, phone, boredom with the virtual environment. These factors can all prevent participants from fully engaging in the task. Although participants with the lowN/highEx profile had significantly higher executive attention control (effortful control score), the stress and distraction may be too overwhelming for them to consistently apply the optimal strategy.

We have speculated in the previous section that one potential reason for low $\mathrm{N} /$ highEx profile's superior performance in late learning of the II task was higher tolerance for frustration and positive reappraisal. We can also speculate that people with the lowN/highEx profile were not only equipped with these tools, their higher levels of effortful control also oriented them towards stronger self-regulation and more goal-directed behaviours (Pereira et al., 2020; Rueda, 2012). Although effortful control was removed in the reduced model in all regression models across the two category learning tasks, this may be due to effortful control being a weaker predictor than affective temperament in predicting the same variances in performance accuracy. effortful control was significantly and negatively correlated with negative affect among participants in this study, and this pattern was also seen in McKinney et al. (2020). We can see that even though effortful control was a separate temperament factor, it was not independent from the affective temperament factors. Higher effortful control ensured that people with the lowN/highEx profile can maintain motivation and positive reappraisal, despite this factor did not independently predict additional proportion of variances.

Effects of Working Memory. Baddeley (2012) suggested that working memory is composed of executive attention control and storage. Executive attention was discussed in the previous section and here the focus is on working memory span, or the number of items/chunks that a person can reliably maintain in their working memory. Working memory span was measured through an RSpan task which was composed of a processing task and a memory recall task. Inclusion of a processing task ensured that the recall task measured the working memory capacity rather than simple short-term memory (Whitney et al., 2001). Scores on the RSpan task did not differ between the two affective temperament profiles in both CR and II tasks, and it was not a significant predictor of performance accuracy in the CR task. Interestingly, in the early stage of II task learning, scores on the RSpan taks were the only significant predictor of accuracy. This shows that people with a higher working memory span learn the II task faster. Similarly, in late learning of II task, Rspan scores accounted for additional variances in accuracy, on top of the variances being predicted by affective temperament. Results from the II task consistently argue that working memory facilitated learning throughout the II task. Taken together the whole picture, higher RSpan scores facilitated II task learning but not CR task learning, contradicting previous studies that 
suggest working memory is only related to rule-based tasks (McDaniel et al., 2014; Smith et al., 2014; Waldron \& Ashby, 2001). Results partially supported prior studies that claim that higher working memory is facilitatory to both rule-based and similarity-based tasks (Kalish et al., 2017; Lewandowsky, 2011). Furthermore, as speculated in the previous section, the CR task used in this study may be too difficult so that everybody was performing badly, thus no effect of either temperament or working memory can be detected.

\section{Limitations}

Results of this study should be interpreted with the following limitations in mind. First, the overall low accuracy and low percentage of participants using CR strategy by the end of the CR task suggests that the optimal decision boundaries of this task were difficult for people to learn. Future studies should separate the category clusters further apart to avoid the floor effect seen in this study. Second, we did not measure participants' current mood state and stress level, these may or may not have changed the affective temperament effects. Despite that the ATQ accurately measures participant's temperament traits, current mood states may still sway their processing scope or cognitive reflexibility. Future studies should include a simple mood questionnaire which can help partialling out the influence of current mood states in studies of affective traits. Third, in both the CR and the II tasks, participants' last block performance was generally not their best-performing block, suggesting that participants may have experienced a fatigue effect and stopped trying to learn the categories.. Future studies may want to use a trials-to-criterion design rather than having everybody completing the entire task: this should provide a better measure of participants' learning speed and strategy transition.

\section{Conclusions}

In summary, the current study shows that people with higher positive affect (lower negative affect) temperament have an advantage in learning similarity-based categorization tasks. Working memory span, as measured by the RSpan task, contributes significantly to II task learning. We did not observe a relationship between affective temperament and learning of the CR tasks. The whole picture of the results suggest that affective temperament factors can be a predictor of people's performance and strategy use on category learning tasks, but only when the tasks have a similarity-based structure. 


\section{References}

Ahadi, S. A., \& Rothbart, M. K. (1994). Temperament, development, and the Big Five. The Developing Structure of Temperament and Personality from Infancy to Adulthood, 189207.

https://books.google.com/books?hl=en\&lr=\&id=bn-OAwAAQBAJ\&oi=fnd\&pg=PA189\&dq=ahadi+roth bart+1994\&ots=8B_5-CgB8X\&sig=xETNoVPWtOLRKQcA5MrQjAOwQFs

Akbari Chermahini, S., \& Hommel, B. (2012). Creative mood swings: divergent and convergent thinking affect mood in opposite ways. Psychological Research, 76(5), 634-640.

Allport, G. W. (1937). Personality: a psychological interpretation. 588. https://psycnet.apa.org/fulltext/1938-01964-000.pdf

Anderson, J. R., \& Betz, J. (2001). A hybrid model of categorization. Psychonomic Bulletin \& Review, $8(4), 629-647$.

Aschkenasy, J. R., \& Odom, R. D. (1982). Classification and perceptual development: exploring issues about integrality and differential sensitivity. Journal of Experimental Child Psychology, 34(3), 435-448.

Ashby, F. G., Alfonso-Reese, L. A., Turken, A. U., \& Waldron, E. M. (1998). A neuropsychological theory of multiple systems in category learning. Psychological Review, 105(3), 442-481.

Ashby, F. G., Ennis, J. M., \& Spiering, B. J. (2007). A neurobiological theory of automaticity in perceptual categorization. Psychological Review, 114(3), 632-656.

Ashby, F. G., \& Gott, R. E. (1988). Decision rules in the perception and categorization of multidimensional stimuli. Journal of Experimental Psychology. Learning, Memory, and Cognition, 14(1), 33-53.

Ashby, F. G., Isen, A. M., \& Turken, A. U. (1999). A neuropsychological theory of positive affect and its influence on cognition. Psychological Review, 106(3), 529-550.

Ashby, F. G., \& Lee, W. W. (1991). Predicting similarity and categorization from identification. Journal of Experimental Psychology. General, 120(2), 150-172.

Ashby, F. G., \& Maddox, W. T. (1990). Integrating information from separable psychological dimensions. Journal of Experimental Psychology. Human Perception and Performance, 16(3), 598-612.

Ashby, F. G., \& Maddox, W. T. (1992). Complex decision rules in categorization: Contrasting novice and experienced performance. Journal of Experimental Psychology. Human Perception and Performance, 18(1), 50-71.

Ashby, F. G., \& Maddox, W. T. (1993). Relations between Prototype, Exemplar, and Decision Bound Models of Categorization. In Journal of Mathematical Psychology (Vol. 37, Issue 3, pp. 372-400). https://doi.org/10.1006/jmps.1993.1023

Ashby, F. G., Maddox, W. T., \& Bohil, C. J. (2002). Observational versus feedback training in rule-based and information-integration category learning. Memory \& Cognition, 30(5), 666-677.

Ashby, F. G., Noble, S., Filoteo, J. V., Waldron, E. M., \& Ell, S. W. (2003). Category learning deficits in Parkinson's disease. Neuropsychology, 17(1), 115-124.

Ashby, F. G., \& Todd Maddox, W. (2005). Human Category Learning. In Annual Review of Psychology (Vol. 56, Issue 1, pp. 149-178). https://doi.org/10.1146/annurev.psych.56.091103.070217

Ashby, F. G., \& Townsend, J. T. (1986). Varieties of perceptual independence. Psychological Review, 93(2), 154-179.

Ashby, F. G., \& Waldron, E. M. (1999). On the nature of implicit categorization. Psychonomic Bulletin \& Review, 6(3), 363-378.

Baddeley, A. (2012). Working memory: theories, models, and controversies. Annual Review of Psychology, 63, 1-29. 
Basso, M. R., Schefft, B. K., Ris, M. D., \& Dember, W. N. (1996). Mood and global-local visual processing. Journal of the International Neuropsychological Society: JINS, 2(3), 249-255.

Bless, H., Fiedler, K., \& Forgas, J. P. (2006). Mood and the regulation of information processing and behavior. Affect in Social Thinking and Behavior, 6584. https://books.google.com/books?hl=en\&lr=\&id=QRfDmLrp1_YC\&oi=fnd\&pg=PA65\&dq=Mood+a nd+the+regulation+of+information+processing+and+behavior\&ots=0XtuoAUlum\&sig=dwA5KOj3 QBKpMvcZpjRxIQUXCgw

Bodenhausen, G. V., Kramer, G. P., \& Süsser, K. (1994). Happiness and stereotypic thinking in social judgment. Journal of Personality and Social Psychology, 66(4), 621-632.

Bopp, K. L., \& Verhaeghen, P. (2005). Aging and verbal memory span: a meta-analysis. The Journals of Gerontology. Series B, Psychological Sciences and Social Sciences, 60(5), P223-P233.

Bott, L., \& Heit, E. (2004). Nonmonotonic Extrapolation in Function Learning. In Journal of Experimental Psychology: Learning, Memory, and Cognition (Vol. 30, Issue 1, pp. 38-50). https://doi.org/10.1037/0278-7393.30.1.38

Brooks, L. R. (1978). Nonanalytic Concept Formation and Memory for Instances. In E. Rosch \& B. Lloyd (Eds.), Cognition and Categorization (pp. 3-170). Lawrence Elbaum Associates.

Clark, M. S., \& Isen, A. M. (1982). Toward understanding the relationship between feeling states and social behavior. Cognitive Social Psychology, 73, 108.

Clore, G. L. (1992). Cognitive phenomenology: Feelings and the construction of judgment. The Construction of Social Judgments, 10, 133-163.

Corbetta, M., Miezin, F. M., Dobmeyer, S., Shulman, G. L., \& Petersen, S. E. (1991). Selective and divided attention during visual discriminations of shape, color, and speed: functional anatomy by positron emission tomography. The Journal of Neuroscience: The Official Journal of the Society for Neuroscience, 11(8), 2383-2402.

Daneman, M., \& Carpenter, P. A. (1980). Individual differences in working memory and reading. Journal of Memory and Language, 19(4), 450.

Davidson, D. J., Zacks, R. T., \& Williams, C. C. (2003). Stroop interference, practice, and aging. Neuropsychology, Development, and Cognition. Section B, Aging, Neuropsychology and Cognition, 10(2), 85-98.

Deng, W. S., \& Sloutsky, V. M. (2016). Selective attention, diffused attention, and the development of categorization. Cognitive Psychology, 91, 24-62.

Derryberry, D., \& Reed, M. A. (1994). Temperament and attention: orienting toward and away from positive and negative signals. Journal of Personality and Social Psychology, 66(6), 1128-1139.

Dienes, B. P. A. (1996). Mood as information: Affective cues for cognitive processing styles. University of Illinois at Urbana-Champaign.

Donkin, C., Newell, B. R., Kalish, M., Dunn, J. C., \& Nosofsky, R. M. (2015). Identifying strategy use in category learning tasks: a case for more diagnostic data and models. Journal of Experimental Psychology. Learning, Memory, and Cognition, 41(4), 933-948.

Easterbrook, J. A. (1959). The effect of emotion on cue utilization and the organization of behavior. In Psychological Review (Vol. 66, Issue 3, pp. 183-201). https://doi.org/10.1037/h0047707

Engle, R. W., \& Kane, M. J. (2004). Executive Attention, Working Memory Capacity, and a Two-Factor Theory of Cognitive Control. In B. H. Ross (Ed.), The psychology of learning and motivation: Advances in research and theory, Vol (Vol. 44, pp. 145-199). Elsevier Science, $x$.

Erickson, M. A. (2008). Executive attention and task switching in category learning: evidence for stimulus-dependent representation. Memory \& Cognition, 36(4), 749-761.

Erickson, M. A., \& Kruschke, J. K. (1998). Rules and exemplars in category learning. Journal of Experimental Psychology. General, 127(2), 107-140.

Estes, W. K. (1986). Array models for category learning. Cognitive Psychology, 18(4), 500-549. 
Evans, D. E., \& Rothbart, M. K. (2007). Developing a model for adult temperament. Journal of Research in Personality, 41(4), 868-888.

Eysenck, M. W., Derakshan, N., Santos, R., \& Calvo, M. G. (2007). Anxiety and cognitive performance: attentional control theory. Emotion , 7(2), 336-353.

Fiedler, K. (2001). Affective influences on social information processing. In J. P. Forgas (Ed.), Handbook of affect and social cognition , (pp (Vol. 457, pp. 163-185). Mahwah, NJ, US.

Folkman, S., \& Moskowitz, J. T. (2000). Positive affect and the other side of coping. The American Psychologist, 55(6), 647-654.

Fredrickson, B. (2009). Positivity. Three Rivers Press.

Fredrickson, B. L., \& Branigan, C. (2005). Positive emotions broaden the scope of attention and thought-action repertoires. Cognition and Emotion, 19(3), 313-332.

Gable, P. A., \& Harmon-Jones, E. (2008). Approach-Motivated Positive Affect Reduces Breadth of Attention. In Psychological Science (Vol. 19, Issue 5, pp. 476-482). https://doi.org/10.1111/j.1467-9280.2008.02112.x

Gable, P., \& Harmon-Jones, E. (2010). The motivational dimensional model of affect: Implications for breadth of attention, memory, and cognitive categorisation. Cognition and Emotion, 24(2), 322-337.

Gabrieli, J. (1995). 14 Contribution of the Basal Ganglia to Skill Learning and Working Memory in Humans. Models of Information Processing in the Basal Ganglia, 277.

Gasper, K. (1999). How thought and emotional awareness influence the role of affect in processing: When attempts to be reasonable fail. Unpublished Doctoral Dissertation, University of Illinois, Urbana-Champaign.

Gasper, K., \& Clore, G. L. (2002). Attending to the big picture: mood and global versus local processing of visual information. Psychological Science, 13(1), 34-40.

Gholson, B., Levine, M., \& Phillips, S. (1972). Hypotheses, strategies, and stereotypes in discrimination learning. Journal of Experimental Child Psychology, 13(3), 423-446.

Goldman-Rakic, P. S. (1995). Toward a circuit model of working memory and the guidance of voluntary motor action. Models of Information Processing in the Basal Ganglia., 382, 131-148.

Goldman-Rakic, P. S. (1995). Cellular basis of working memory. Neuron, 14(3), 477-485.

Goldsmith, H. H., Buss, A. H., Plomin, R., Rothbart, M. K., Thomas, A., Chess, S., Hinde, R. A., \& McCall, R. B. (1987). Roundtable: what is temperament? Four approaches. Child Development, 58(2), 505-529.

Gorlick, M. A., \& Maddox, W. T. (2013). Priming for performance: valence of emotional primes interact with dissociable prototype learning systems. PloS One, 8(4), e60748.

Hanania, R., \& Smith, L. B. (2009). Selective attention and attention switching: towards a unified developmental approach. In Developmental Science (Vol. 13, Issue 4, pp. 622-635). https://doi.org/10.1111/j.1467-7687.2009.00921.x

Hatala, R. M., Brooks, L. R., \& Norman, G. R. (2003). Practice makes perfect: the critical role of mixed practice in the acquisition of ECG interpretation skills. Advances in Health Sciences Education: Theory and Practice, 8(1), 17-26.

Hayes-Roth, F. (1985). Rule-based systems. Communications of the ACM. https://dl.acm.org/doi/abs/10.1145/4284.4286?casa_token=yn4nTTlqugQAAAAA:9yTp8nthxzsb 0AGPfo9wLrkmH9YPz5sIYRS3wF-BdkE4zb1yjUgN-cBfPbABQ5p0zpKzxGtqbesqOg

Hertel, G., \& Fiedler, K. (1994). Affective and cognitive influences in social dilemma game. In European Journal of Social Psychology (Vol. 24, Issue 1, pp. 131-145).

https://doi.org/10.1002/ejsp.2420240110 
Homa, D., Sterling, S., \& Trepel, L. (1981). Limitations of exemplar-based generalization and the abstraction of categorical information. Journal of Experimental Psychology. Human Learning and Memory, 7(6), 418-439.

Isbell, L. M. (1999). Beyond heuristic information processing: Systematic processing in happy and sad moods [University of Illinois at Urbana-Champaign]. https://www.ideals.illinois.edu/handle/2142/82288

Isen, A. M. (1970). Success, failure, attention, and reaction to others: The warm glow of success. Journal of Personality and Social Psychology, 15(4), 294-301.

Isen, A. M. (1987). Positive Affect, Cognitive Processes, and Social Behavior. In L. Berkowitz (Ed.), Advances in Experimental Social Psychology (Vol. 20, pp. 203-253). Academic Press.

Isen, A. M., \& Daubman, K. A. (1984). The influence of affect on categorization. Journal of Personality and Social Psychology, 47(6), 1206-1217.

Ji, L.-J., Yap, S., Best, M. W., \& McGeorge, K. (2019). Global Processing Makes People Happier Than Local Processing. Frontiers in Psychology, 10, 670.

Kalish, M. L., Newell, B. R., \& Dunn, J. C. (2017). More is generally better: Higher working memory capacity does not impair perceptual category learning. Journal of Experimental Psychology. Learning, Memory, and Cognition, 43(4), 503-514.

Kemler, D. G. (1978). Patterns of hypothesis testing in children's discriminative learning: A study of the development of problem-solving strategies. In Developmental Psychology (Vol. 14, Issue 6, pp. 653-673). https://doi.org/10.1037/0012-1649.14.6.653

Kimchi, R. (1992). Primacy of wholistic processing and global/local paradigm: a critical review. Psychological Bulletin, 112(1), 24-38.

Konishi, S., Karwazu, M., Uchida, I., Kikyo, H., Asakura, I., \& Miyashita, Y. (1999). Contribution of Working Memory to Transient Activation in Human Inferior Prefrontal Cortex during Performance of the Wisconsin Card Sorting Test. In Cerebral Cortex (Vol. 9, Issue 7, pp. 745-753). https://doi.org/10.1093/cercor/9.7.745

Lewandowsky, S. (2011). Working memory capacity and categorization: individual differences and modeling. Journal of Experimental Psychology. Learning, Memory, and Cognition, 37(3), 720-738.

Lewandowsky, S., Yang, L.-X., Newell, B. R., \& Kalish, M. L. (2012). Working memory does not dissociate between different perceptual categorization tasks. Journal of Experimental Psychology. Learning, Memory, and Cognition, 38(4), 881-904.

Lombardi, W. J., Andreason, P. J., Sirocco, K. Y., Rio, D. E., Gross, R. E., Umhau, J. C., \& Hommer, D. W. (1999). Wisconsin Card Sorting Test performance following head injury: dorsolateral fronto-striatal circuit activity predicts perseveration. Journal of Clinical and Experimental Neuropsychology, 21(1), 2-16.

Maddox, W. T., Ashby, F. G., \& Bohil, C. J. (2003). Delayed feedback effects on rule-based and information-integration category learning. Journal of Experimental Psychology. Learning, Memory, and Cognition, 29(4), 650-662.

Maddox, W. T., Chandrasekaran, B., Smayda, K., Yi, H.-G., Koslov, S., \& Beevers, C. G. (2014). Elevated depressive symptoms enhance reflexive but not reflective auditory category learning. Cortex; $a$ Journal Devoted to the Study of the Nervous System and Behavior, 58, 186-198.

Maddox, W. T., Pacheco, J., Reeves, M., Zhu, B., \& Schnyer, D. M. (2010). Rule-based and information-integration category learning in normal aging. Neuropsychologia, 48(10), 2998-3008.

May, C. P., \& Hasher, L. (1998). Synchrony effects in inhibitory control over thought and action. Journal of Experimental Psychology. Human Perception and Performance, 24(2), 363-379.

McDaniel, M. A., Cahill, M. J., Robbins, M., \& Wiener, C. (2014). Individual differences in learning and transfer: stable tendencies for learning exemplars versus abstracting rules. Journal of Experimental Psychology. General, 143(2), 668-693. 
McKinney, C., Stearns, M., \& Szkody, E. (2020). Temperament and Affective and Behavioral ODD Problems in Emerging Adults: Moderation by Gender and Perceived Parental Psychopathology. Child Psychiatry and Human Development. https://doi.org/10.1007/s10578-020-00969-8

Medin, D. L., \& Schaffer, M. M. (1978). Context theory of classification learning. Psychological Review, 85(3), 207-238.

Miles, S. J., \& Minda, J. P. (2009). Learning new categories: Adults tend to use rules while children sometimes rely on family resemblance. Proceedings of the Annual Meeting of the Cognitive Science Society, 31. https://escholarship.org/content/qt0hc8x4kk/qt0hc8x4kk.pdf

Miller, R., \& Wickens, J. R. (1991). Corticostriatal cell assemblies in selective attention and in representation of predictable and controllable events. Concepts in Neuroscience, 2(1), 65-95.

Minda, J. P., Desroches, A. S., \& Church, B. A. (2008). Learning rule-described and non-rule-described categories: a comparison of children and adults. Journal of Experimental Psychology. Learning, Memory, and Cognition, 34(6), 1518-1533.

Minda, J. P., \& Smith, J. D. (2001). Prototypes in category learning: the effects of category size, category structure, and stimulus complexity. Journal of Experimental Psychology. Learning, Memory, and Cognition, 27(3), 775-799.

Murphy, G. L., Bosch, D. A., \& Kim, S. (2017). Do Americans Have a Preference for Rule-Based Classification? Cognitive Science, 41(8), 2026-2052.

Navon, D. (1977). Forest before trees: The precedence of global features in visual perception. Cognitive Psychology, 9(3), 353-383.

Nielsen, E., \& Minda, J. P. (2018). The relationship between depressive symptoms, hypomanic symptoms, mood, and category learning. https://doi.org/10.31234/osf.io/yqxtn

Noseworthy, T. J., \& Goode, M. R. (2011). Contrasting rule-based and similarity-based category learning: The effects of mood and prior knowledge on ambiguous categorization. Journal of Consumer Psychology: The Official Journal of the Society for Consumer Psychology, 21(3), 362-371.

Nosofsky, R. M. (1986). Attention, similarity, and the identification-categorization relationship. Journal of Experimental Psychology. General, 115(1), 39-57.

Owen, A. M., Roberts, A. C., Hodges, J. R., Summers, B. A., Polkey, C. E., \& Robbins, T. W. (1993). Contrasting mechanisms of impaired attentional set-shifting in patients with frontal lobe damage or Parkinson's disease. Brain: A Journal of Neurology, 116 ( Pt 5), 1159-1175.

Park, D. C., Lautenschlager, G., Hedden, T., Davidson, N. S., Smith, A. D., \& Smith, P. K. (2002). Models of visuospatial and verbal memory across the adult life span. Psychology and Aging, 17(2), 299-320.

Peirce, J. W. (2007). PsychoPy-Psychophysics software in Python. Journal of Neuroscience Methods, 162(1), 8-13.

Pelley, M. E. L., Le Pelley, M. E., Newell, B. R., \& Nosofsky, R. M. (2019). Deferred Feedback Does Not Dissociate Implicit and Explicit Category-Learning Systems: Commentary on Smith et al. (2014). In Psychological Science (p. 095679761984126). https://doi.org/10.1177/0956797619841264

Pereira, E. J., Gurguryan, L., \& Ristic, J. (2020). Trait-Level Variability in Attention Modulates Mind Wandering and Academic Achievement. Frontiers in Psychology, 11, 909.

Pickford, R. W., Eysenck, H. J., \& Notcutt, B. (1954). The structure of human personality. The British Journal of Sociology, 5(2), 183.

Plude, D. J., Enns, J. T., \& Brodeur, D. (1994). The development of selective attention: a life-span overview. Acta Psychologica, 86(2-3), 227-272.

Posner, M. I., \& Petersen, S. E. (1990). The attention system of the human brain. Annual Review of Neuroscience, 13, 25-42. 
Posner, M. I., \& Rothbart, M. K. (2007). Research on attention networks as a model for the integration of psychological science. Annual Review of Psychology, 58, 1-23.

Posner, M. I., Rothbart, M. K., Sheese, B. E., \& Tang, Y. (2007). The anterior cingulate gyrus and the mechanism of self-regulation. Cognitive, Affective \& Behavioral Neuroscience, 7(4), 391-395.

Rabi, R., \& Minda, J. P. (2014). Rule-Based Category Learning in Children: The Role of Age and Executive Functioning. In PLOS ONE (Vol. 9, Issue 1, p. e85316). https://doi.org/10.1371/journal.pone.0085316

Rabi, R., \& Minda, J. P. (2016). Category learning in older adulthood: A study of the Shepard, Hovland, and Jenkins (1961) tasks. Psychology and Aging, 31(2), 185-197.

Racine, C. A., Barch, D. M., Braver, T. S., \& Noelle, D. C. (2006). The effect of age on rule-based category learning. Neuropsychology, Development, and Cognition. Section B, Aging, Neuropsychology and Cognition, 13(3-4), 411-434.

Rao, S. M., Bobholz, J. A., Hammeke, T. A., Rosen, A. C., Woodley, S. J., Cunningham, J. M., Cox, R. W., Stein, E. A., \& Binder, J. R. (1997). Functional MRI evidence for subcortical participation in conceptual reasoning skills. Neuroreport, 8(8), 1987-1993.

R Core Team. (2020). R: a language and environment for statistical computing. $R$ Foundation for Statistical Computing, Vienna, Austria.

Reed, S. K. (1972). Pattern recognition and categorization. Cognitive Psychology, 3(3), 382-407.

Robert, C., Borella, E., Fagot, D., Lecerf, T., \& de Ribaupierre, A. (2009). Working memory and inhibitory control across the life span: Intrusion errors in the Reading Span Test. Memory \& Cognition, 37(3), 336-345.

Rogers, R. D., Andrews, T. C., Grasby, P. M., Brooks, D. J., \& Robbins, T. W. (2000). Contrasting cortical and subcortical activations produced by attentional-set shifting and reversal learning in humans. Journal of Cognitive Neuroscience, 12(1), 142-162.

Rothbart, M., \& Bates, J. (Eds.). (2007). Temperament. In Handbook of Child Psychology (Vol. 37, p. 1111). John Wiley \& Sons, Inc.

Rothbart, M. K. (2012). Advances in temperament. Handbook of Temperament, 3-20.

Rothbart, M. K., Ahadi, S. A., \& Evans, D. E. (2000). Temperament and personality: origins and outcomes. Journal of Personality and Social Psychology, 78(1), 122-135.

Rothbart, M. K., Derryberry, D., \& Posner, M. I. (1994). A psychobiological approach to the development of temperament. https://psycnet.apa.org/record/1994-97632-003

Rothbart, M. K., \& Rueda, M. R. (2005). The development of effortful control. https://psycnet.apa.org/record/2005-03029-009

Rueda, M. R. (2012). Effortful control. In M. Zentner (Ed.), Handbook of temperament, (pp (Vol. 750, pp. 145-167). The Guilford Press, xvii.

Schwarz, N. (1990). Happy but mindless? Mood effects on problem solving and persuasion. Handbook of Motivation and Cognition, 2, 527-561.

Schwarz, N., \& Clore, G. L. (1983). Mood, misattribution, and judgments of well-being: Informative and directive functions of affective states. Journal of Personality and Social Psychology, 45(3), 513-523.

Searston, R. A., \& Tangen, J. M. (2017). The Emergence of Perceptual Expertise with Fingerprints Over Time. Journal of Applied Research in Memory and Cognition, 6(4), 442-451.

Sinclair, R. C., Mark, M. M., \& Clore, G. L. (1994). Mood-Related Persuasion Depends on (Mis)Attributions. Social Cognition, 12(4), 309-326.

Sloman, S. A. (1996). The empirical case for two systems of reasoning. Psychological Bulletin, 119(1), 3-22. 
Smith, J. D., Boomer, J., Zakrzewski, A. C., Roeder, J. L., Church, B. A., \& Ashby, F. G. (2014). Deferred feedback sharply dissociates implicit and explicit category learning. Psychological Science, 25(2), 447-457.

Volz, H.-P., Gaser, C., Häger, F., Rzanny, R., Mentzel, H.-J., Kreitschmann-Andermahr, I., Kaiser, W. A., \& Sauer, H. (1997). Brain activation during cognitive stimulation with the Wisconsin Card Sorting Test-a functional MRI study on healthy volunteers and schizophrenics. Psychiatry Research: Neuroimaging, 75(3), 145-157.

Waldron, E. M., \& Gregory Ashby, F. (2001). The effects of concurrent task interference on category learning: Evidence for multiple category learning systems. In Psychonomic Bulletin \& Review (Vol. 8, Issue 1, pp. 168-176). https://doi.org/10.3758/bf03196154

West, R. (1999). Age differences in lapses of intention in the Stroop task. The Journals of Gerontology. Series B, Psychological Sciences and Social Sciences, 54(1), P34-P43.

Whitney, P., Arnett, P. A., Driver, A., \& Budd, D. (2001). Measuring central executive functioning: what's in a reading span? Brain and Cognition, 45(1), 1-14.

Whittle, S., Allen, N. B., Fornito, A., Lubman, D. I., Simmons, J. G., Pantelis, C., \& Yücel, M. (2009). Variations in cortical folding patterns are related to individual differences in temperament. Psychiatry Research, 172(1), 68-74.

Whittle, S., Yap, M. B. H., Yücel, M., Fornito, A., Simmons, J. G., Barrett, A., Sheeber, L., \& Allen, N. B. (2008). Prefrontal and amygdala volumes are related to adolescents' affective behaviors during parent-adolescent interactions. Proceedings of the National Academy of Sciences of the United States of America, 105(9), 3652-3657.

Williams, B. R., Ponesse, J. S., Schachar, R. J., Logan, G. D., \& Tannock, R. (1999). Development of inhibitory control across the life span. Developmental Psychology, 35(1), 205-213.

Wyer, R. S., Jr, Clore, G. L., \& Isbell, L. M. (1999). Affect and information processing. Advances in Experimental Social Psychology.

https://www.sciencedirect.com/science/article/pii/S0065260108602713 